\title{
Cuidado multiprofissional em saúde de crianças e adolescentes com diabetes: estudo da literatura
}

RESUMO | Objetivo: descrever o cuidado multiprofissional em saúde para as crianças e adolescentes com Diabetes Mellitus. Método: revisão integrativa, de caráter descritivo e qualitativo. Realizado na Scientific Electronic Library Online (SciELO) e Google acadêmico, com a coleta de dados em junho de 2021. Mediante critérios de inclusão e exclusão e cruzamento dos descritores, foram selecionadas 8 publicações sobre o tema. Os resultados foram expostos em dois quadros. Resultados e discussões: Prevaleceram artigos dos anos de 2020 sobre o tema. A maioria das revistas publicadas são da área da enfermagem. Educar em saúde, torna-se essencial no acompanhamento e manutenção da diabetes. O adoecimento das crianças e adolescentes com DM, repercute em toda a família. Conclusão: A abordagem multiprofissional é extremamente importante, por cuidar da criança/ adolescente, mas de todos os envolvidos, orientando as intervenções e potencializando o cuidado no domicílio.

Palavras-chaves: Multiprofissional; Criança; Saúde; Diabetes; Cuidado; Adolescente.

ABSTRACT | Objective: to describe the multidisciplinary health care for children and adolescents with Diabetes Mellitus. Method: integrative review, descriptive and qualitative. Carried out at: Scientific Electronic Library Online (SciELO) and academic Google, with data collection in June 2021. Through inclusion and exclusion criteria and crossing of descriptors, 8 publications on the subject were selected. The results were displayed in two tables. Results and discussions: Articles from the 2020s on the subject prevailed. Most journals published are in the field of nursing. Health education becomes essential in monitoring and maintaining diabetes. The illness of children and adolescents with DM affects the whole family. Conclusion: The multidisciplinary approach is extremely important, as it takes care of the child/adolescent, but of all those involved, guiding interventions and enhancing home care.

Keywords: Multiprofesional; Kid; Health; Diabetes; Caution; Teenagers.

RESUMEN | Objetivo: describir la atención de salud multidisciplinaria para niños y adolescentes con Diabetes Mellitus. Método: revisión integradora, descriptiva y cualitativa. Realizado en: Scientific Electronic Library Online (SciELO) y Google académico, con recolección de datos en junio de 2021. Mediante criterios de inclusión y exclusión y cruce de descriptores, se seleccionaron 8 publicaciones sobre el tema. Los resultados se muestran en dos tablas. Resultados y discusiones: prevalecieron los artículos de la década de 2020 sobre el tema. La mayoría de las revistas publicadas pertenecen al campo de la enfermería. La educación sanitaria se vuelve fundamental para controlar y mantener la diabetes. La enfermedad de los niños y adolescentes con DM afecta a toda la familia. Conclusión: El abordaje multidisciplinario es de suma importancia, ya que se ocupa del niño / adolescente, pero de todos los involucrados, orientando las intervenciones y potenciando la atención domiciliaria.

Palabras claves: Multiprofessional; Niño; Salud; Diabetes; Precaución; Adolescente.

\section{Kátia Cristina Barbosa Ferreira}

Enfermeira. Universidade Estadual da Paraíba. ORCID: 0000-0002-3353-2973

\section{Maria Clara Ribeiro Costa}

Enfermeira. Centro Universitário de João Pessoa, Paraíba.

ORCID: 0000-0003-4422-3719

\section{Luana Gomes Leitão Rodrigues}

Enfermeira. Centro Universitário de João Pessoa, Paraíba.

ORCID: 0000-0001-5259-1357

\section{Lúcia Gomes de Souza Silva}

Enfermeira. Faculdade Maurício de Nassau. ORCID: 0000-0001-6998-435X

\section{Luciene de Souza Santos Albuquerque}

Enfermeira. Centro Universitário de João Pessoa. ORCID: 0000-0001-5119-195X

\section{Raeuna Gabrielly Barros da Costa}

Fisioterapeuta. Universidade Estadual da Paraíba ORCID: 0000-0003-4501-73119
Recebido em: 16/06/2021

Aprovado em: 05/07/2021

\section{INTRODUÇÃO}

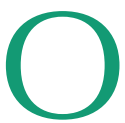
Diabetes Mellitus (DM) é uma patologia metabólica, que se classifica mediante a ausência completa ou parcial da produção de insulina. No mundo, existem três tipos de DM: a DM tipo I, DM tipo II e DM gestacional, cada uma com algumas peculiaridades diferentes, sendo a primeira mais frequente na fase infanto-juvenil. Na DM tipo I, ocorre a ausência absoluta da produção de insulina pelo pâncreas, em contrapartida, na DM tipo Il existe uma produção, mas em menor quantidade, diferenciando uma da outra. Na DM gestacional, os níveis glicêmicos ficam alterados durante a gestação, e também voltam ao normal após esse pe- ríodo gravídico, porém, em alguns casos, podem permanecer e se tornar a DM do tipo II, de forma crônica ${ }^{(1)}$.

Crianças e adolescentes necessitam de um controle e acompanhamento, o cuidado acontece desde o diagnóstico, quando se realiza o rastreamento da DM na infância ou adolescência, visando minimizar agravos e complicações para a saúde dessa população. Diante do diagnóstico, as crianças e adolescentes precisam ser acompanhados por uma equipe multiprofissional, que cuide e oriente sobre o uso de insulinas e sua administração, percepção dos sinais de hipo e hiperglicemia, alimentação ideal, organização da rotina, prática de atividade física, autocuidado e terapias ${ }^{(1-2)}$.

Os familiares também devem ser orientados quanto aos cuidados, e precisam fazer parte do acompanhamento multiprofissional, mediante as orienta- 
ções realizadas. A equipe multiprofissional precisa prepara a família também, exercendo a integralidade do cuidado, e o compartilhamento de ações ${ }^{(1-2)}$.

Assim, estudos têm demonstrado que as mães de crianças com DM1 sentem-se incompetentes diante do diagnóstico e despreparadas para lidar com a situação complexa da doença, manifestando sentimentos de ansiedade, estresse e isolamento social ${ }^{(1-2)}$. A crianças que possuem a DM, precisam de suporte, no qual é necessário a criatividade da equipe de saúde, ofertando o cuidado de forma lúdica, usando de brinquedos terapêuticos, teatro, músicas e histórias para amenizar o medo e inseguranças da família junto a criança ${ }^{(2)}$.

A continuidade do cuidado é algo primordial a discutir quando a temática se refere a doenças crônicas, pois essas crianças e adolescentes precisam ser acoIhidas em todos os serviços de saúde, desde o primário até o terciário, e em níveis ambulatoriais também ${ }^{(3)}$. Desse modo, foi possível construir a questão de pesquisa deste estudo: Como se dá o cuidado multiprofissional em saúde para crianças/ adolescentes com diabetes mellitus na literatura? Tem-se por objetivo: descrever o cuidado multiprofissional em saúde para as crianças e adolescentes com Diabetes Mellitus.

\section{MÉTODO}

Estudo de revisão integrativa, de caráter descritivo e qualitativo. Realizado nos meios eletrônicos: Scientific Electronic Library Online (SciELO) e Google acadêmico, com o período de coleta de dados em junho de 2021. Para a seleção dos artigos, foram organizados os critérios de inclusão e exclusão, como os critérios de inclusão foram: publicações em português, completas, gratuitas e direcionadas ao objetivo, além de serem publicados entre os anos de 2016 e 2021. E como critérios de exclusão: publicações em outros idiomas, fora do período estabelecidos, incompletas e que cobrem taxas de acesso ao material.

Para selecionar as publicações foi desenvolvido um instrumento, elaborado pelos autores do artigos, que buscava colher informações importantes, como: autores, título, ano de publicação, periódico, nível de evidência e os principais cuidados multiprofissionais oferecidos para as crianças e adolescentes com DM. Para realizar a busca, definiu-se alguns Descritores em Ciências da Saú- de (DeCS): Multiprofissional; Criança; Saúde; Diabetes; Cuidado e Adolescente, conforme o cruzamento do operador booleano AND.

A busca ocorreu a partir do encontro de 105 publicações sobre o tema, no primeiro momento. Em seguida, foi inserido o período de publicação, ficando 58 publicações para seguir na análise. Foi realizada a leitura dos títulos das 58 publicações, no qual apenas 08 encontravam-se na perspectiva deste estudo. Houve a leitura dos resumos das 08 publicações, e todas respondiam ao objetivo do estudo. Por fim, realizou-se a leitura integral dos materiais, verificando que as 08 publicações realmente poderiam ser inseridas neste estudo. Ao final, houve a exclusão de 97 publicações e inclusão de 08 publicações. Todo o processo foi validado por dois pesquisadores relacionados a temática do estudo.

\section{RESULTADOS}

As publicações foram organizadas em dois quadros, com o auxílio do programa da Microsoft Word, no qual foram expostas as informações que caracterizam os materiais encontrados da melhor forma.

\section{QUADRO 1 - Apresentação das publicações referentes aos cuidados multiprofissionais para crianças e adolescentes com DM:}

\begin{tabular}{|c|c|c|c|}
\hline Autores & Título & Ano de publicação & Periódico \\
\hline QUEIROZ, M.V.O. et al. ${ }^{(4)}$. & $\begin{array}{l}\text { Sensibilizando a criança com diabetes para o } \\
\text { cuidado de si: Contribuição à prática educativa. }\end{array}$ & 2016. & Escola de Enfermagem Anna Nery. \\
\hline WOLKERS, P.C.B. et al. ${ }^{(5)}$. & $\begin{array}{l}\text { Crianças com diabetes mellitus tipo 1: acesso aos } \\
\text { imunobiológicos especiais e à puericultura. }\end{array}$ & 2017. & Revista da Escola de Enfermagem da USP. \\
\hline ALMEIDA, K.O. et al. ${ }^{(7)}$. & $\begin{array}{l}\text { Cuidados direcionados ás Práticas de Educação } \\
\text { Física Escolar para Crianças com Diabetes Tipo I. }\end{array}$ & 2020. & Brazilian Journal of Technology. \\
\hline
\end{tabular}




\begin{tabular}{|l|c|c|c|}
\hline SILVA, L.C.S. de. et al. ${ }^{(9)}$. & $\begin{array}{c}\text { Cintura Hipertrigliceridêmica e Fatores Associa- } \\
\text { dos em Crianças e Adolescentes Portadores de } \\
\text { Diabetes Melito Tipo 1. }\end{array}$ & 2020. & Revista Paulista de Pediatria. \\
\hline HERMES, T.S.V. et al. ${ }^{(10)}$. & $\begin{array}{c}\text { Criança diabética do tipo 1 e o convívio familiar: } \\
\text { repercussões no manejo da doença. }\end{array}$ & 2018. & Saúde e Debate. \\
\hline Fonte: Dados da pesquisa, 2021.
\end{tabular}

Prevaleceram artigos dos anos de 2020, e nos anos de 2016, 2017, 2018 e 2019, publicaram apenas um artigo sobre o tema. A maioria das revistas publicadas são da área da enfermagem, no qual faz-se pertinente explicar, que o enfermeiro é um dos profissionais mais ativos no cuidado a criança e adolescente com DM.

Os títulos das publicações enunciam a grandiosidade da temática, no qual percebe-se, que vários pontos importantes são elencados em cada abordagem de títulos.

\section{DISCUSSÃo}

Educar em saúde, como visto em um dos estudos, torna-se essencial no acompanhamento e manutenção da diabetes.
Diante disso, é imprescindível, que os profissionais de saúde, conheçam a realidade do paciente com DM, priorizem as necessidades dele, objetivando ampliar a visão das condutas terapêuticas multiprofissionais ${ }^{(4)}$.

A partir do que se conhece sobre DM, os profissionais podem ajudar a família e os cuidadores, moldando o que se precisa para se obter o sucesso no cuidado. Não se pode desvalorizar as experiências anteriores da família, existem problemáticas que cercam o cuidado da criança e do adolescente, como: autocuidado, hábitos familiares, déficit no acompanhamento da equipe multiprofissional, não adesão do tratamento e outros problemas ${ }^{(4-11-12)}$.

Levando em consideração a realidade da criança/adolescente, insere-se os determinantes sociais, que abrangem o cuidado em saúde, analisando o cuidado da família com a criança, o dia-a-dia, fatores sociais e econômicos que interlaçam a criança, estrutura familiar, fatores biológicos e etc. $\mathrm{O}$ acesso aos medicamentos para tratamento da DM ainda é um desafio no Brasil, diante da desigualdade em saúde, algumas crianças e adolescentes não dão continuidade ao tratamento, devido a barreiras no acesso à saúde, ferindo o princípio da universalidade. Por este motivo, o cuidado multiprofissional e interinstitucional em saúde é importante, para diminuir as fragilidades do sistema e garantir o acesso para o tratamento continuado da $\mathrm{DM}^{(3)}$.

QUADRO 2 - Descrição dos cuidados multiprofissionais para crianças e adolescentes com DM encontrados nas publicações:

\begin{tabular}{|c|c|}
\hline Autores & O Cuidado Multiprofissional para Crianças e Adolescentes com Diabetes Mellitus \\
\hline QUEIROZ, M.V.O. et al. ${ }^{(4)}$. & - A educação em saúde para a família e cuidadores é uma prática multiprofissional. \\
\hline WOLKERS, P.C.B. et al. ${ }^{(3)}$. & $\begin{array}{l}\text { - Os profissionais de saúde que cuidam das crianças nesta condição crônica, levam em consideração dos determinan- } \\
\text { tes sociais, as vulnerabilidades e todo o contexto que a criança vive. }\end{array}$ \\
\hline WOLKERS, P.C.B. et al. ${ }^{(5)}$. & $\begin{array}{l}\text { - } 0 \text { estudo apontou várias problemáticas sobre a vacinação das crianças com DM: esquemas vacinais incompletos, } \\
\text { reforços anuais atrasados, ausência do cartão de vacinação nos atendimentos, desinformação sobre o caráter especial } \\
\text { da vacinação, bem como a ênfase na necessidade de apresentar formulário específico para obter a vacinação. Estas } \\
\text { problemáticas são orientações multiprofissionais, pois na consulta de acompanhamento, deve-se orientar os familiares } \\
\text { para a realização da vacinação. }\end{array}$ \\
\hline SOUZA, R.R. et al. ${ }^{(6)}$. & $\begin{array}{l}\text { - Os familiares e cuidadores demonstraram algumas dificuldades neste estudo, referente ao cuidado com: aplicação } \\
\text { correta da insulina, às restriçõ̂es alimentares e à ausência de apoio por parte da escola, no controle alimentar e } \\
\text { tratamento medicamentoso. }\end{array}$ \\
\hline ALMEIDA, K.O. et al. ${ }^{(7)}$. & $\begin{array}{l}\text { - Na educação física, as crianças precisam ser orientadas e avaliadas continuamente para a prática de exercícios } \\
\text { físicos. } \\
\text { - É importante observar sinais de hipoglicemia, e observar as especificidades da criança/adolescente. }\end{array}$ \\
\hline DIXE, M.A.C.R. et al. ${ }^{(8)}$. & $\begin{array}{l}\text {-Os educadores escolares também fazem parte do acompanhamento multiprofissional de crianças e adolescentes com } \\
\text { DM, por isso, precisam conhecer sobre a doença, e auxiliar nos cuidados com a criança no ambiente escolar. }\end{array}$ \\
\hline SILVA, L.C.S. de. et al. ${ }^{(9)}$. & $\begin{array}{l}\text { - } 0 \text { nutricionista também faz parte ativamente dos cuidados com as crianças e adolescentes com DM, neste artigo, no } \\
\text { que se refere a cintura hipertrigliceridêmica. Que é uma ferramenta para ajudar no diagnóstico de síndrome metabóli- } \\
\text { ca, tornando-se um rastreador importante. }\end{array}$ \\
\hline HERMES, T.S.V. et al. ${ }^{(10)}$. & $\begin{array}{l}\text { - Um dos resultados importantes do artigo, é que os profissionais de saúde precisam auxiliar os familiares no manejo } \\
\text { e na rotina das crianças e adolescentes com DM, para que eles possam aderir ao tratamento. }\end{array}$ \\
\hline
\end{tabular}


A criança/adolescente com DM 1, necessita de avaliações com regularidade, sendo importante toda a rede de apoio envolvida no cuidado com ela. Infelizmente, nos serviços de saúde nem sempre isso é possível, o acesso ao atendimento primário é uma grande problemática, gerando internações em grande escala e complicações da DM com facilidade, o que poderia ser evitado com acompanhamento correto $^{(5)}$. Como visto nos resultados, a vacinação dessas crianças e adolescentes também é um problema a ser repensado, existem déficits nos indicadores de imunização dessas crianças, deixando-as susceptíveis ao acometimento de outras doenças, além da $\mathrm{DM}^{(5)}$.

$\mathrm{O}$ adoecimento das crianças e adolescentes com DM, repercute em toda a família, não é só a criança/adolescente que adoece, a família também se preocupa, e ela como rede de apoio precisa estar fortalecida, para não gerar sofrimento de um para o outro ${ }^{(10-12)}$. Neste sentido, a abordagem multiprofissional é extremamente importante, por cuidar da criança/ adolescente, mas de todos os envolvidos, orientando as intervenções e potencializando o cuidado no domicílio. É importante incentivar a criança/adolescente com DM quanto ao estilo de vida, mudanças na rotina, manejo da insula, realização de exercícios físicos, verificação da glicemia capilar e outros cuidados ${ }^{(6)}$.

Conforme visto neste artigo, a ativida- de física é importante, e tem sido implementada de forma contínua no cuidado ao portador de DM, junto ao tratamento de insulinoterapia e medicamentoso, ajudando na prevenção e controle da DM de forma significativa, aumentando a qualidade de vida dos pacientes ${ }^{(7)}$.

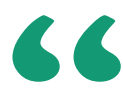

Não é fácil controlar os níveis de glicemia dos pacientes com DM, a todo momento a equipe multiprofissional precisa estar perto da família, avaliando riscos e agravos.

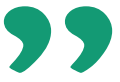

Não é fácil controlar os níveis de glicemia dos pacientes com DM, a todo momento a equipe multiprofissional precisa estar perto da família, avaliando riscos e agravos. Sendo assim, o nutricionista também é importante, por ajudar no controle metabólico de forma individualizada ${ }^{(9)}$. Foi visto, que a escola também é importante, por ser um dos ambientes mais visitados pelas crianças/adolescentes em seu dia-a-dia, sendo interessante, que os educadores conheçam sobre a doença e falem sobre os cuidados no processo escolar, auxiliando a família e a equipe de saúde ${ }^{(8)}$.

\section{CONCLUSÃO}

O estudo alcançou o seu objetivo, e conseguiu expor sobre o cuidado multiprofissional para crianças e adolescentes com DM. Percebeu-se, que a DM 1 é predominante na faixa etária infanto-juvenil, e que necessita de cuidados de todos os profissionais de saúde, tais como: educadores físicos, enfermeiros, médicos, dentistas, nutricionistas, psicólogos e os professores da escola que frequentam.

Recomenda-se este estudo para os acadêmicos de saúde, profissionais da área, familiares e todos os interessantes em compreender mais sobre a doença e o cuidado multiprofissional. Necessita-se de novas pesquisas sobre o tema, para que o assunto seja ampliando na comunidade acadêmica. Os profissionais de saúde exercem uma função primordial, cuidando desde a prevenção até os agravos e complicações que podem ocorrer na DM. Entendeu-se, que a família é rede de apoio, e que precisa fazer parte do cuidado em saúde, sendo inserida no que for pertinente.

\section{Referências}

1. Okido, ACC. et al. La demanda de cuidado de los niños con diabetes mellitus tipo 1. Esc. Anna Nery 21 (2), 2017.

2. Pennafort, VPS dos. et al. Brinquedo terapêutico instrucional no cuidado cultural da criança com diabetes tipo 1. Rev. Bras. Enferm. 71 (suppl 3), 2018.

3. Wolkers, PCB. et al. Crianças com diabetes mellitus tipo 1: vulnerabilidade, cuidado e acesso à saúde. Texto contexto - enferm. 28, 2019.

4. Queiroz MVO, Brito LMMC, Pennafort VPS, Bezerra FSM. Sensibilizando a criança com diabetes para o cuidado de si: Contribuição à prática educativa. Esc Anna Nery 2016;20(2):337-343.

5. Wolkers, PCB. et al. Children with type 1 Diabetes Mellitus: access to special immunobiological and child care. Rev. esc. enferm. USP vol.51, 2017.

6. Souza RR, Marquete VF, Vieira VCL, Fischer MJB, Spigolon DN, Marcon SS. Cuidando da diabetes no âmbito domiciliar. Rev enferm UERJ, Rio de Janeiro, 2020; 28:e46013.

7. Almeida, KO de. et al. Care directed to school physical education practices for children with type I diabetes. Braz. J. Technol., Curitiba, v. 3, n. 3, p. 103-114, jul./set. 2020.

8. Dixe, MACR de. et al. Efeitos de um programa de educação nos conhecimentos e na autopercepção dos educadores escolares na preparação para cuidar de crianças diabéticas tipo 1. Einstein (São Paulo) 18, 2020.

9. Silva, LCS de. et al. Cintura hipertrigliceridêmica e fatores associados em crianças e adolescentes portadores de diabetes melito tipo 1. Rev. paul. pediatr. 38 , 2020.

10. Hermes, TSV. et al. Criança diabética do tipo 1 e o convívio familiar: repercussões no manejo da doença. Saúde debate 42 (119), 2018.

11. Sardinha, AHI de , Souza, AS, Cavalcante, MRMota. Nursing (Säo Paulo) ; 21(238): 2080-2084, mar. 2018

12. Ortiz L de OM, Damião EBC, Rossato LM, Alves RCP. Melhores práticas de enfermagem em educação em diabetes à criança hospitalizada: uma revisão integrativa. Rev. Eletr. Enferm. 2017, 19. 